\title{
Sublingual Piroxicam for Relief of Pain during Diagnostic Hysteroscopy a Randomized Controlled Trial
}

\author{
El-Said N1, Shalakany $A^{1}$, Abdelrahem $A^{2}$, Elbohoty ${ }^{1 *}$, Tharwat $A^{1}$ \\ and Kotb $\mathbf{A}^{1}$ \\ ${ }^{1}$ Department of Obstetrics and Gynecology, Ain Shams University, Egypt \\ ${ }^{2}$ Department of Obstetrics and Gynecology, Biadiah Central Hospital, Egypt
}

\section{Research Article \\ Volume 2 Special Issue 1}

Received Date: December 06, 2016

Published Date: January 06, 2017

*Corresponding author: Ahmed Elbohoty, Assistant Professor of Obstetrics and Gynecology, Faculty of Medicine, Ain Shams University, Egypt, Email: elbohoty79@gmail.com

\section{Abstract}

Objective: The aim of the current trial was to evaluate the efficacy of sublingual piroxicam prior to office hysteroscopy in reducing the procedure-related pain.

Methods: The current placebo-controlled randomized clinical trial was conducted at the Early Cancer Detection Unit (ECDU) at Ain Shams University Maternity Hospital. The study included women planned to undergo office (diagnostic) hysteroscopy for various indications. The recruited women were randomized into one of two groups: group I, including women who received piroxicam $20 \mathrm{mg}$ sublingual tablets; and group II, including women who received an antacid/antiflatulent tablet (as a placebo) containing calcium carbonate $420 \mathrm{mg}$ plus dimethicone $10 \mathrm{mg}$. The primary outcome was pain. Pain was assessed using a 10-cm visual analogue scale. Secondary outcomes included the procedure duration, rate of failed procedure, the level of difficulty of the procedure, as well as, the patient's acceptability.

Results: The mean age of included women was 34.8 \pm 9.5 years (range: 22-37 years). The median 10-cm VAS for pain was significantly lower in women of group I [Piroxicam Group] [median (IQR): 4 (3-7) vs. 5 (4-8), respectively, p=0.031]. The proportion of women who had moderate/severe pain was lower, but not to a significant level, in women of group I. There were no significant differences between women of both groups regarding the procedure duration, as well as, for the rates of failed or difficult procedure.

Conclusion: Sublingual piroxicam prior to office hysteroscopy seems to be significantly associated with less procedurerelated pain, yet with no significant effect on the procedure duration, or the rates of failed or difficult procedure.

Keywords: Office hysteroscopy; Pain; Piroxicam 


\section{Introduction}

Hysteroscopy has become a relatively common diagnostic tool in current gynecological practice, particularly during assessment of abnormal uterine bleeding and infertility [1]. The main features of value that advantages hysteroscopy are both the high diagnostic yield and the relatively less invasive approach [2]. The 'office' approach, i.e. performing the procedure in the outpatient clinic without need for hospital admission or receiving anesthesia, augments these features [3]. The main problem with this 'office' approach is the pain [4]. Several strategies have been proposed over decades to reduce the hysteroscopy procedure-related pain, including smaller calibers [5], vaginoscopic approach (i.e. without vaginal speculum and without grasping the cervix with a tenaculum) [6], and the use of saline as a distending medium [7], pain remains a major problem with office hysteroscopy. In addition, several pharmacological options have been proposed to minimize the procedure-related, including prior administration of misoprostol [8], paracervical block [9], and even the use of narcotic analgesia [10]. The administration of a nonsteroidal anti-inflammatory drug (NSAID) has been also proposed. Naproxen [11], mefanimic acid [12] and ketoprofen [13] have all been tried with variable efficacy. Prioxicam is a potent analgesic of the NSAID group [14]. A pharmaceutical sublingual preparation has been long marketed in Egypt, with relatively easy use, rapid action, and a low side effects profile [15]. The aim of the current trial was to assess the efficacy of sublingual piroxicam tablets administered prior to office hysteroscopy in reducing the procedure-related pain.

\section{Methods}

The current placebo-controlled randomized clinical trial was conducted at the Early Cancer Detection Unit (ECDU) at Ain Shams University Maternity Hospital during the period between January 2012 and October 2012. The study protocol was in agreement to the Declaration of Helsinki for Ethical Medical Research [last updated in Korea, 2008] and was approved by the Ethical Committee of Obstetrics and Gynecology Department, Ain Shams University. All recruited women signed informed written consent after thorough explanation of the purpose and procedure of the study. All approached women had to withdraw from the study or decline without being adversely impacted regarding the medical service they should receive.
The study included women presented to the ECDU and planned to undergo office (diagnostic) hysteroscopy for various indications, including abnormal uterine bleeding, infertility or missed IUCD threads.

Women who had a contraindication to hysteroscopy (e.g. suspected pregnancy, genital tract infection) and those who had a contraindication to piroxicam (known hypersensitivity or peptic ulcer disease) were not included in the study.

\section{Sample size calculation}

To the best of our knowledge there is no preceding trial assessing the role of piroxicam $20 \mathrm{mg}$ sublingual tablets in reducing outpatient hysteroscopy associated pain. We tried to use reasonable number 155 women divided into 2 groups.

\section{Randomization, allocation and intervention}

The recruited women were randomized into one of two groups: group I, including women who received piroxicam $20 \mathrm{mg}$ sublingual tablets [Feldene Flash ${ }^{\circledR}$, Pfizer Pharmaceuticals, Egypt], and group II, including women who received an antacid/anti-flatulent tablet (as a placebo) containing calcium carbonate $420 \mathrm{mg}$ plus dimethicone $10 \mathrm{mg} \quad$ [Glycodal ${ }^{\circledR}, \quad$ Alexandria Pharmaceuticals, Egypt], which is very similar in shape to the treatment tablets used in group I, and rapidly dissolves when taken sublingually. Randomization was performed using a Computer-generated randomization system. The allocated groups were concealed in seriallynumbered sealed opaque envelops that was only opened just after recruitment. Women in both groups received the allocated treatment 30 minutes before the procedure, and were instructed not to take any form of analgesia.

\section{Hysteroscopy procedure}

Normal saline was used as a distension medium. Vaginoscopic approach was adopted for all included women; no speculum was inserted; the cervix was not grasped with a tenaculum. The procedure was performed using a rigid $30^{\circ} \mathrm{Hamou}$ II telescope with a Hopkins II lens system (Karl Stortz ${ }^{\circledR}$, Tuttlingen, Germany). The sheath had a $4.5-\mathrm{mm}$ caliber with a $2.9-\mathrm{mm}$ rod lens. The infusion system used was Hamou Endomat (Karl Stortz ${ }^{\circledR}$, Tuttlingen, Germany). Saline was infused at a rate of 100 $\mathrm{ml} / \mathrm{min}$ with a pressure of $100 \mathrm{~mm} \mathrm{Hg}$. Hysteroscopy procedures were performed by the staff members of the ECDU, with at least 3 years of experience (Figure 1). 


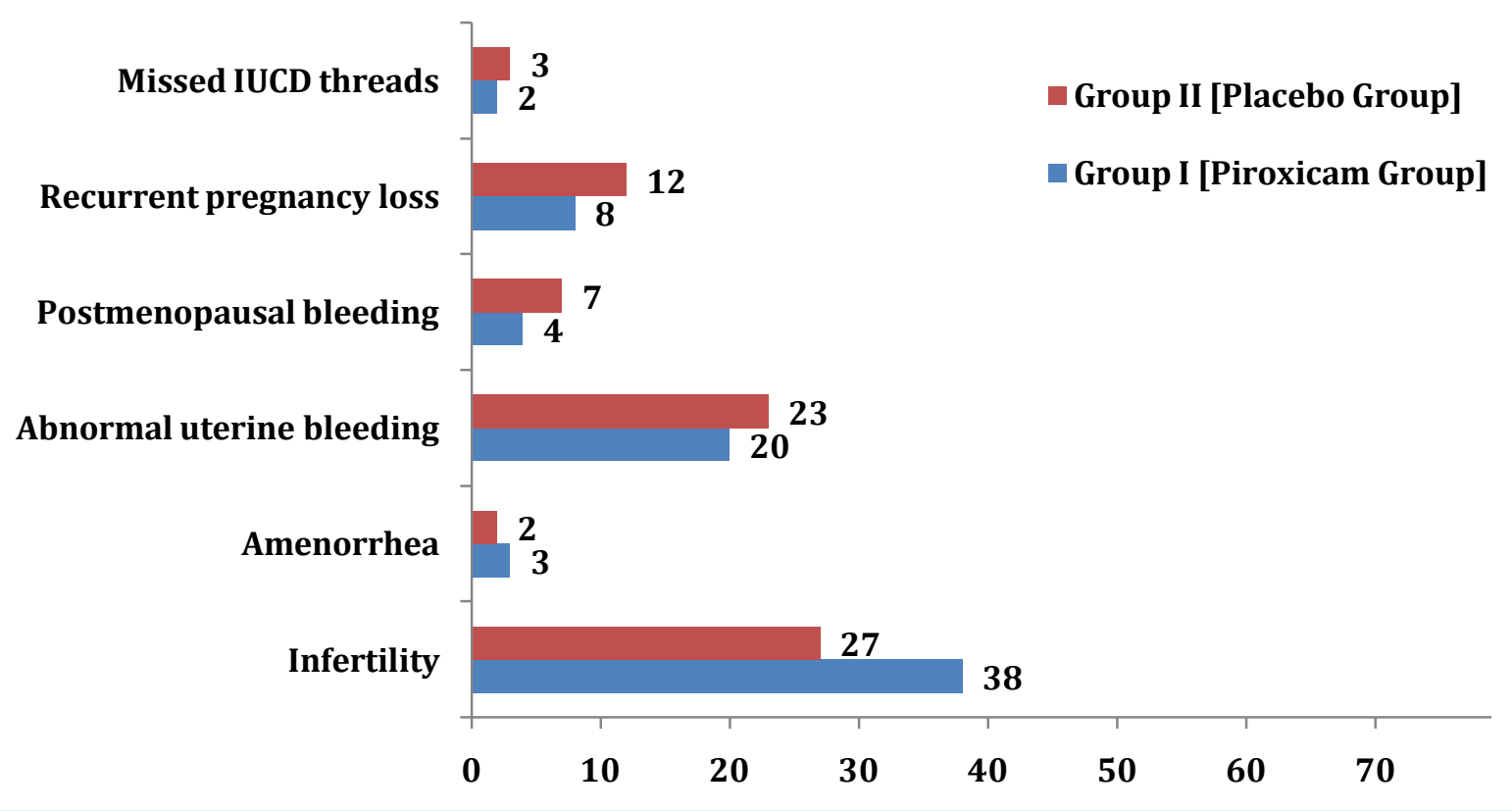

Figure 1: Bar-Chart showing Indication for Office Hysteroscopy in Both Groups.

\section{Study outcomes}

The primary outcome was pain. Pain was assessed using a $10-\mathrm{cm}$ visual analogue scale (Figure 2), with 0 denoting no pain, while 10 denoting the worst pain. Pain was graded as no/mild pain, if the VAS was $\leq 6$; or moderate/severe if the VAS was $\geq 7$. Pain was assessed immediately after the procedure.

Secondary outcomes included the procedure duration, rate of failed procedure, the level of difficulty of the procedure, as well as, the patient's acceptability. The procedure duration was calculated from the time of insertion of the instrument till the time of removal of the scope from the vagina. Failed procedure was defined as any situation in which there was failed access to the uterine cavity or in which the patient's intolerability resulted in interruption of the procedure. The level of difficulty was graded into very easy, easy, equivocal, difficult and failed; as shown in Table 1. The patient's impression about the procedure was labeled as 'acceptable' if she stated that she would accept repeating the procedure under the same conditions, or 'not acceptable' if she stated she would desire receiving anesthesia to undergo the procedure anymore.

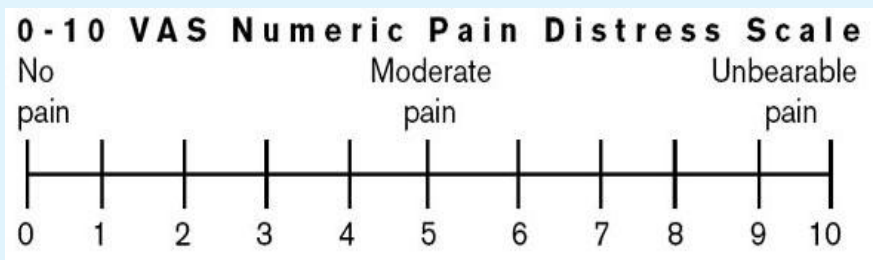

Figure 2: 10-cm Visual Analogue Scale.

\begin{tabular}{|c|c|}
\hline Very easy & $\begin{array}{c}\text { Getting into the uterine cavity with no } \\
\text { resistance or having to withdraw the scope } \\
\text { tip }\end{array}$ \\
\hline Easy & $\begin{array}{c}\text { Having to withdraw and reintroduce the } \\
\text { scope tip once }\end{array}$ \\
\hline Difficult & $\begin{array}{c}\text { Having to withdraw and reintroduce the } \\
\text { scope tip more than once, or resistance at the } \\
\text { internal os }\end{array}$ \\
\hline Failed & $\begin{array}{c}\text { Getting into the uterine cavity with } \\
\text { signicant patient discomfort, but not to the } \\
\text { extent to interrupt the procedure }\end{array}$ \\
\hline $\begin{array}{c}\text { Failed access to the uterine cavity or the } \\
\text { patient's intolerability resulted in } \\
\text { interruption of the procedure }\end{array}$ \\
\hline
\end{tabular}

Table 1: Grading of the Level of Difficulty in Office Hysteroscopy Procedure. 


\section{Statistical methods}

Statistical analysis was performed using SPSS $^{\circledR}$ for Windows version 20. Difference between independent groups was analyzed using independent student's t-test (for numeric parametric variables); Mann-Whitney's Utest (for numeric non-parametric variables); and chisquared test, risk ratio and its 95\% confidence interval (for categorical variables). Yates' continuity correction was applied to the chi-squared test whenever one or more of observed values were less than 5 . Significance level was set at 0.05 . The number needed to treat (NNT) was calculated as the reciprocal of the absolute risk reduction between the two groups.

\section{Results}

A total of 155 women were recruited in the trial; of them 149 were included in the final analysis. Figure 2 shows a flow diagram of the study course and excluded women.

The mean age of included women was $34.8 \pm 9.5$ years (range: 22-37 years). The median parity was 1 (range: 0 $5)$. Of the included women, 10 (6.7\%) women were postmenopausal. There were no significant differences between women of both groups regarding these initial characteristics (Table 2). Figure 3 shows the various indications of office hysteroscopy in included women in both groups. There was no significant difference between both groups regarding these indications.

\begin{tabular}{|c|c|c|c|}
\hline & $\begin{array}{c}\text { Group I } \\
\text { [Piroxicam } \\
\text { Group] } \\
\text { (n=75) }\end{array}$ & $\begin{array}{c}\text { Group II } \\
\text { [Placebo } \\
\text { Group] } \\
\text { (n=74) }\end{array}$ & $\mathbf{P}$ \\
\hline Age (years) & $33.4 \pm 8.1$ & $35.3 \pm 10.1$ & $0.208^{1}$ \\
\hline Parity & $1(0-2)$ & $1(0-2)$ & $0.512^{2}$ \\
\hline Previous CS & $11(14.7 \%)$ & $12(16.2 \%)$ & $0.794^{3}$ \\
\hline $\begin{array}{c}\text { Postmenopausal } \\
\text { Status }\end{array}$ & $3(4 \%)$ & $7(9.5 \%)$ & $0.315^{3}$ \\
\hline
\end{tabular}

Table 2: Initial Characteristics in Both Groups.

Data presented as mean \pm SD; median (interquartile range); or number (percentage)

CS cesarean section

1 Analysis using independent student's t-test

2 Analysis using Mann-Whitney's U-test

3 Analysis using continuity-corrected chi-squared test

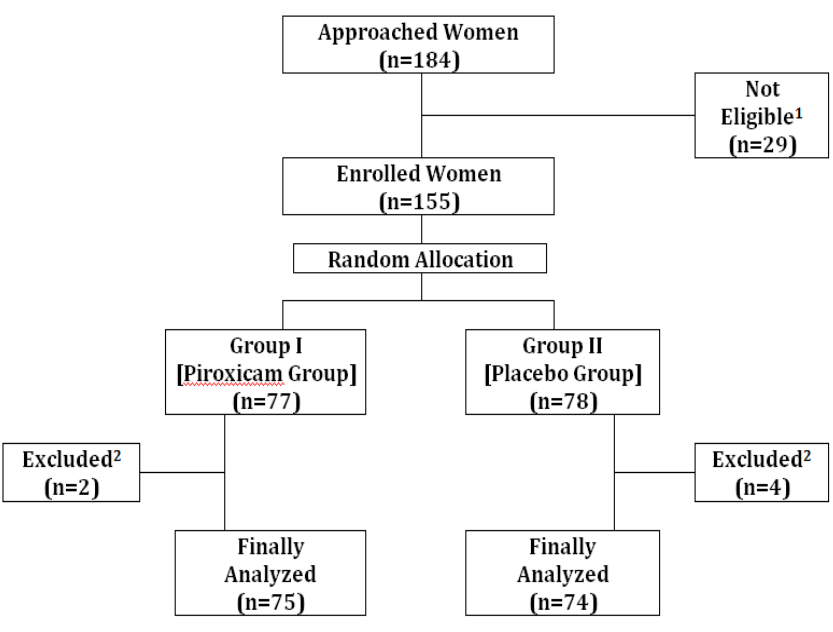

Figure 3: Flow-Diagram showing Study Course.

1 Not Eligible as they do not fulfill the inclusion/exclusion criteria.

2 Excluded from analysis due to abortion of the procedure after receiving the allocated drug, owing to heavy uterine bleeding or purulent vaginal discharge.

The median 10-cm VAS for pain was significantly lower in women of group I [Piroxicam Group] [median (IQR): 4 $(3-7)$ vs. $5(4-8)$, respectively, $p=0.031]$. The proportion of women who had moderate/severe pain was lower, but not to a significant level, in women of group I (Table 3).

\begin{tabular}{|c|c|c|c|c|}
\hline & $\begin{array}{c}\text { Group I } \\
\text { [Piroxicam } \\
\text { Group] } \\
\text { (n=75) }\end{array}$ & $\begin{array}{c}\text { Group } \\
\text { II } \\
\text { [Placeb } \\
\text { o } \\
\text { Group] } \\
\text { (n=74) }\end{array}$ & $\boldsymbol{P}$ & NNT \\
\hline $\begin{array}{c}\text { Pain Score } \\
\text { (10-cm } \\
\text { VAS) }\end{array}$ & $4(3-7)$ & $5(4-8)$ & $\begin{array}{c}0.031 \\
1\end{array}$ & - \\
\hline $\begin{array}{c}\text { Moderate/ } \\
\text { Severe } \\
\text { Pain }\end{array}$ & $17(22.7 \%)$ & $\begin{array}{c}19 \\
(25.7 \% \\
\text { ) }\end{array}$ & $\begin{array}{c}0.668 \\
2\end{array}$ & 33 \\
\hline
\end{tabular}

Table 3: Primary Outcome (Pain) in Both Groups.

Data presented as median (interquartile range); or number (percentage)

VAS visual analogue scale

NNT number needed to treat

1 Analysis using Mann-Whitney's U-test 
2 Analysis using continuity-corrected chi-squared test

There were no significant differences between women of both groups regarding the procedure duration, as well

\begin{tabular}{|c|c|c|c|c|}
\hline & $\begin{array}{c}\text { Group I } \\
\text { [Piroxicam Group] } \\
\text { (n=75) }\end{array}$ & $\begin{array}{c}\text { Group II } \\
\text { [Placebo Group] } \\
\text { (n=74) }\end{array}$ & P & NNT \\
\hline Procedure Duration (min) & $1.96 \pm 0.85$ & $1.94 \pm 0.7$ & $0.876^{1}$ & - \\
\hline Failed Procedure & $1(1.3 \%)$ & $2(2.7 \%)$ & $0.991^{2}$ & 73 \\
\hline Difficult Procedure & $6(8 \%)$ & $11(14.9 \%)$ & 0.2892 & 15 \\
\hline Unacceptable Procedure & $8(10.7 \%)$ & $14(18.9 \%)$ & $0.156^{2}$ & 12 \\
\hline
\end{tabular}

Table 4: Secondary Outcomes in Both Groups.

Data presented as mean $\pm \mathrm{SD}$; or number (percentage)

NNT number needed to treat

1 Analysis using independent student's t-test

2 Analysis using continuity-corrected chi-squared test

The current study showed that sublingual piroxicam (Feldene Flash $®$, Pfizer) was associated with a significantly lower $10-\mathrm{cm}$ VAS for office hysteroscopy procedure-related pain when compared to placebo. However, from the clinical point of view, this treatment has marginal benefit. The NNT for reducing the rates of moderate/severe pain was quite high $(\mathrm{NNT}=33)$, and so were the NNTs for the rates of failed, difficult or unacceptable procedures $[\mathrm{NNT}=73,15$ and 12 , respectively].

These results were in some agreement with an early study conducted by Nagele et al. who administered mefanimic acid (500 mg) $60 \mathrm{~min}$ prior to diagnostic hysteroscopy and showed no significant benefit in the discomfort experienced during the procedure [16].

Similarly, in a recent well-designed double-blind randomized controlled trial, rectal NSAID (diclofenac sodium) was compared to vaginal misoprostol and to placebo in infertile nulliparous women (whom should be assumed to have a narrower cervix). The authors found no benefit of rectal NSAID or vaginal misoprostol over the placebo, regarding the procedure-related pain or discomfort [12].

In a similar study to the latter one, Issat et al. compared vaginal misoprostol to rectal ketoprofen to placebo in office hysteroscopy procedures. They, however, administered the treatments 4 hours before the as, for the rates of failed, difficult or unacceptable procedure (Table 4). 
on the procedure duration, or the rates of failed or difficult procedure; a finding that makes it no different from previously studies NSAIDs.

\section{References}

1. van Dongen $\mathrm{H}$, de Kroon $\mathrm{CD}$, Jacobi $\mathrm{CE}$, Trimbos JB, Jansen FW (2007) Diagnostic hysteroscopy in abnormal uterine bleeding: a systematic review and meta-analysis. BJOG 114(6): 664-675.

2. van Dongen $\mathrm{H}$, Timmermans A, Jacobi CE, Elskamp T, de Kroon CD, et al. (2011) Diagnostic hysteroscopy and saline infusion sonograp hy in the diagnosis of intrauterine abnormalities: an assessment of patient preference. Gynecol Surg 8(1): 65-70.

3. Siristatidis C, Chrelias C, Salamalekis G, Kassanos D (2010) Office hysteroscopy: current trends and potential applications: a critical review. Arch Gynecol Obstet 282(4): 383-388.

4. Kuzel D, Tóth D, Hrazdírová L, Mára M, Fucíková Z (2006) Office hysteroscopy-state of the art. Ceska Gynekol 71(3): 220-225.

5. Török P, Major T (2013) Evaluating the level of pain during office hysteroscopy according to menopausal status, parity, and size of instrument. Arch Gynecol Obstet 287(5): 985-988.

6. Cooper NA, Smith P, Khan KS, Clark TJ (2010) Vaginoscopic approach to outpatient hysteroscopy: a systematic review of the effect on pain. BJOG 117(5): 532-539.

7. Cooper NA, Smith P, Khan KS, Clark TJ (2011) A systematic review of the effect of the distension medium on pain during outpatient hysteroscopy. Fertil Steril 95(1): 264-271.

8. Tam WH, Yuen PM (2001) Use of diclofenac as an analgesic in outpatient hysteroscopy: a randomized, double-blind, placebo-controlled study. Fertil Steril 76(5): 1070-1072.

9. Sharma JB, Aruna J, Kumar P, Roy KK, Malhotra N, et al. (2009) Comparison of efficacy of oral drotaverine plus mefenamic acid with paracervical block and with intravenous sedation for pain relief during hysteroscopy and endometrial biopsy. Indian J Med Sci 63(6): 244-252.
10. Koçum A, Sener M, Izmirli H, Haydardedeoğlu B, Arıboğan A (2014) Efficacy of intravenous dexketoprofen trometamol compared to intravenous paracetamol for postoperative pain management after day-case operative hysteroscopy: randomized, double-blind, placebo-controlled study. Agri 26(1): 15-22.

11. van EE R, Hemrika DJ, van der Linden CT (1993) Pain relief following day-case diagnostic hysteroscopylaparoscopy for infertility: a double-blind randomized trial with preoperative naproxen versus placebo. Obstet Gynecol 82(6): 951-954.

12. Nagele F, Lockwood G, Magos AL (1997) Randomised placebo controlled trial of mefenamic acid for premedication at outpatient hysteroscopy: a pilot study. Br J Obstet Gynaecol 104(7): 842-844.

13. Issat T, Beta J, Nowicka MA, Maciejewski T, Jakimiuk AJ (2014) A randomized, single blind, placebocontrolled trial for the pain reduction during the outpatient hysteroscopy after ketoprofen or intravaginal misoprostol. J Minim Invasive Gynecol 21(5): 921-927.

14. Xu S, Rouzer CA, Marnett LJ (2014) Oxicams, a class of nonsteroidal anti-inflammatory drugs and beyond. IUBMB Life 66(12): 803-811.

15. Gramke HF, Petry JJ, Durieux ME, Mustaki JP, Vercauteren M, et al. (2006) Sublingual piroxicam for postoperative analgesia: preoperative versus postoperative administration: a randomized, doubleblind study. Anesth Analg 102(3): 755-758.

16. Hassa H, Aydin Y, Oge T, Cicek K (2013) Effectiveness of vaginal misoprostol and rectal nonsteroidal antiinflammatory drug in vaginoscopic diagnostic outpatient hysteroscopy in primarily infertile women: double-blind, randomized, controlled trial. J Minim Invasive Gynecol 20(6): 880-885.

17. Teran-Alonso MJ, De Santiago J, Usandizaga $\mathrm{R}$, Zapardiel I (2014) Evaluation of pain in office hysteroscopy with prior analgesic medication: a prospective randomized study. Eur J Obstet Gynecol Reprod Biol 178: 123-127.

18. Trindade PA, Giglio FP, Colombini-Ishikiriama BL, Calvo AM, Modena KC, et al. (2012) Sublingual ketorolac and sublingual piroxicam are equally effective for postoperative pain, trismus, and swelling 
management in lower third molar removal. Oral Surg Oral Med Oral Pathol Oral Radiol 114(1): 27-34.

19. KandaSwamy GV, Dhanasekaran AK, Elangovan A, John B, Viswaroop B, et al. (2015) Randomized double blinded placebo controlled trial comparing diclofenac and piroxicam in management of acute renal colic and its clinical implications. Urol J 12(2): 2069-2073.

20. Ravishankar K, Tayade H, Mandlik R (2011) Sublingual piroxicam in migraine without aura. J Assoc Physicians India 59: 494-497. 